\section{Glukokortikoide keine Gefahr für Knochen}

\author{
Die Langzeitgabe höherer Dosen oraler Glukokortikoide kann bei \\ postmenopausalen Frauen eine Osteoporose induzieren. Doch wie \\ sieht es mit inhalativen Glukokortikoiden aus?
}

\begin{abstract}
$\mathrm{N}$ achdem inhalative Glukokortikoide seit knapp 20 Jahren verfügbar sind, findet man mittlerweile viele Patienten, die über längere Zeit mit den Lokaltherapeutika behandelt worden sind. Schwedische Forscher verglichen die Knochendichte postmenopausaler Frauen, die längerfristig inhalative Glukokortikoide (IG, $n=106$ ) erhalten hatten, mit der von altersentsprechenden Frauen ohne Glukokortikoide $(n=674)$. Dokumentiert wurden außerdem die
\end{abstract}

Daten von 49 Frauen, die orale und/ oder intraartikuläre Glukokortikoide zusätzlich zu den inhalativen Substanzen erhalten hatten.

IG (überwiegend Budesonid, Beclometason und Fluticason) wurden im Durchschnitt 8,2 $\pm 5,03$ Jahre in einer Dosierung von $853 \mu \mathrm{g} / \mathrm{d}$ angewandt. Die Knochendichte der Frauen mit IG unterschied sich insgesamt nicht signifikant von der der Kontrollfrauen $\left(0,434 \mathrm{~g} / \mathrm{cm}^{2}\right.$ vs. $0,429 \mathrm{~g} / \mathrm{cm}^{2}$ ). Dagegen war die Kno- chendichte bei den Frauen mit einer zusätzlichen oralen/intraartikulären Applikation deutlich erniedrigt $\left(0,408 \mathrm{~g} / \mathrm{cm}^{2}\right)$. Die 23\% der Probandinnen, die mehr als $1.000 \mu \mathrm{g}$ eines inhalativen Glukokortikoids pro Tag applizierten, hatten eine statistisch jedoch nicht signifikante - niedrigere Knochenmasse als die Frauen der IG-Gruppe mit geringeren Tagesdosen.

Fazit: Die Verwendung inhalativer Glukokortikoide über einen längeren Zeitraum hinweg hat keinen relevanten Einfluss auf die Knochendichte postmenopausaler Frauen.
Elmståhl $S$ et al. Is there an association between inhaled corticosteroids and bone density in postmenopausal women? J Allergy Clin Immunol 2003; 111: 91-6

\title{
Asthmakontrolle: Selbst ist der Patient!
}

\section{Bei der Asthmatherapie ist neben der Kontrolle von Entzündung und Symptomen die Verbesserung der Lebensqualität entschei- dend. Kann dieses Ziel nur durch engmaschige ärztliche Betreuung erreicht werden, oder kann der Patient auch selbst seinen Beitrag dazu leisten? Eine niederländische Studie gibt Antwort.}

$E_{\mathrm{i}}^{\mathrm{i}}$ in Forscherteam der Universität Nijmegen teilte 214 Patienten unmittelbar nach ihrer Asthma-Erstdiagnose zwei Gruppen zu: 104 Patienten erhielten eine konventionelle Behandlung entsprechend den allgemeinen

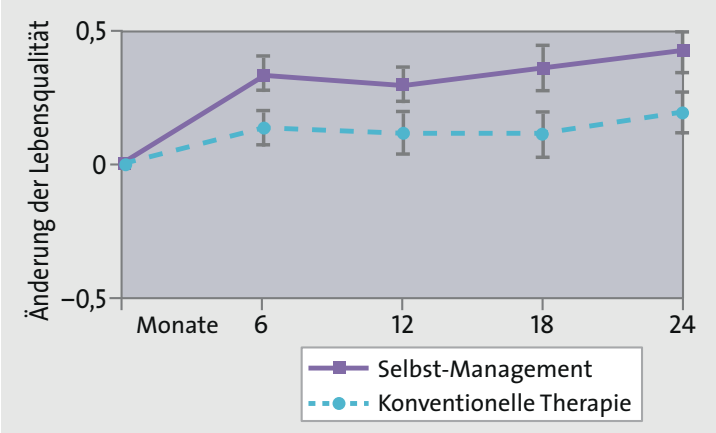

Veränderung der Lebensqualität, gemessen anhand des Asthma Quality of Life Questionnaire, von Patienten mit Selbstmanagement-Schulung und konventionell behandelten Patienten
Therapierichtlinien mit einer einmaligen Einweisung in das Medikationsschema und die Inhalationstechnik sowie der Aufforderung zu Arztbesuchen alle drei bis sechs Monate. 110 Patienten nahmen an einem Selbstmanagement-Trainingsprogramm mit vier Lehreinheiten teil, in denen vor allem die selbstständige Anpassung der Medikamentendosis in Abhängigkeit von Symptomatik und Peak-Flow-Werten vermittelt wurde. Diese Patienten sollten zweimal jährlich zu Kontrolluntersuchungen erscheinen.

Im anschließenden Beobachtungszeitraum schafften es die Selbstmanagement-Patienten (SMPatienten), 81 von insgesamt 105 Wochen erfolgreich zu absolvieren, den konventionell behandelten $\mathrm{Pa}$ tienten gelang dies nur in 74 von
103 Wochen $(p=0,003)$. Eine Woche war als erfolgreich definiert, wenn der Dyspnoe-Index über dem Median des gesamten Behandlungszeitraumes lag. Die Werte im Asthma Quality of Life Questionnaire steigerten sich in der SMGruppe um 0,21 Punkte pro Arztbesuch im Vergleich zu 0,10 Punkten bei den Kontrollpatienten $(p=0,055)$. Vor allem aber verringerte sich bei den SM-Patienten das Gefühl von Unsicherheit und Hilflosigkeit gegenüber der Krankheit. FEV 1 -Werte und die Histamindosis im Provokationstest änderten sich nicht. Pro Patient konnten bei den instruierten Asthmatikern 217 Hübe eines inhalativen Glukokortikoids im Vergleich zu den Kontrollen eingespart werden $(\mathrm{p}<0,05)$.

Fazit: Patienten profitieren von einer Asthmaschulung, bei der sie lernen, ihre Glukokortikoiddosis symptomorientiert nach einem vorgegebenen Therapieplan selbst festzulegen. Bei guter Krankheitskontrolle und insgesamt niedrigerer Glukokortikoiddosis steigt vor allem die Lebensqualität. $\quad b k$

Thoonen BP et al. Self-management of asthma in general practice, asthma control and quality of life: a randomised controlled trial. Thorax 2003; 58: 30-6 